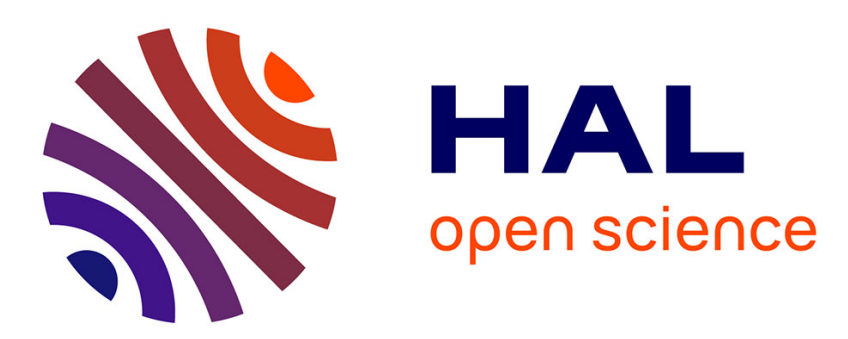

\title{
La qualité du lait a-t-elle un prix? Une estimation hédonique de la valeur des attributs spécifiques du lait de vache
}

Dominique Desbois

\section{- To cite this version:}

Dominique Desbois. La qualité du lait a-t-elle un prix? Une estimation hédonique de la valeur des attributs spécifiques du lait de vache. Cahiers d'Economie et de Sociologie Rurales, 2015, 96 (03), pp.499 - 529. 10.4074/S1966960715003057 . hal-01608256

\section{HAL Id: hal-01608256 \\ https://hal.science/hal-01608256}

Submitted on 27 May 2020

HAL is a multi-disciplinary open access archive for the deposit and dissemination of scientific research documents, whether they are published or not. The documents may come from teaching and research institutions in France or abroad, or from public or private research centers.
L'archive ouverte pluridisciplinaire HAL, est destinée au dépôt et à la diffusion de documents scientifiques de niveau recherche, publiés ou non, émanant des établissements d'enseignement et de recherche français ou étrangers, des laboratoires publics ou privés.

\section{(ㅇ)(1) $\$$}

Distributed under a Creative Commons Attribution - NonCommercial - NoDerivatives| 4.0 


\section{Revue d'Études en Agriculture et Environnement}

http://www.necplus.eu/RAE

Additional services for Revue d'Études en Agriculture et Environnement:

Email alerts: $\underline{\text { Click here }}$

Subscriptions: $\underline{\text { Click here }}$

Commercial reprints: Click here

Terms of use : $\underline{\text { Click here }}$

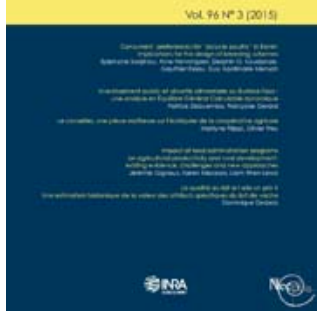

\section{La qualité du lait a-t-elle un prix ? Une estimation hédonique de la valeur des attributs spécifiques du lait de vache}

Dominique Desbois

Revue d’Études en Agriculture et Environnement / Volume 96 / Issue 03 / September 2015, pp 499 -

529

DOI: 10.4074/S1966960715003057, Published online: 30 December 2015

Link to this article: http://www.necplus.eu/abstract S1966960715003057

How to cite this article:

Dominique Desbois (2015). La qualité du lait a-t-elle un prix ? Une estimation hédonique de la valeur des attributs spécifiques du lait de vache. Revue d'Études en Agriculture et Environnement, 96, pp 499-529 doi:10.4074/S1966960715003057

Request Permissions : $\underline{\text { Click here }}$ 


\title{
La qualité du lait a-t-elle un prix? Une estimation hédonique de la valeur des attributs spécifiques du lait de vache
}

\section{Dominique DESBOIS}

INRA, UMR0210 Économie Publique, F-78850 Thiverval-Grignon, France e-mail:dominique.desbois@agroparistech.fr ; desbois@grignon.inra.fr

Résumé - Dans le contexte d'une fin programmée du système des quotas laitiers, la valorisation du lait est une variable clé de la rentabilité des exploitations laitières. Le lait de vache tire sa valeur des différents produits de sa transformation. Les signes d'origine et de qualité constituent un facteur majeur de différenciation pour les spécialités fromagères mais aussi pour le beurre et la crème. Ainsi, la valorisation du lait dépend de sa composition mais aussi d'attributs qualitatifs du produit laitier ayant trait à son origine et sa typicité. Basé sur le Réseau d'Information Comptable Agricole (RICA), ce travail a donc pour objectif d'estimer la valeur implicite pour le producteur des attributs quantitatifs et qualitatifs du lait au moyen d'une méthodologie de régression hédonique grâce à des données de panel dans une période de forte variation du prix de ce produit (2006-2008). Sur cette base, nous donnons des estimations des prix implicites de la matière grasse et de la matière protéique, ainsi que la valorisation des différents signes officiels et professionnels de qualité compte tenu des contextes régionaux de production et de transformation étudiés.

Mots-clés : qualité du lait, régression hédonique, signes de qualité, Réseau d'Information Comptable Agricole, France

\section{Is there a price for milk quality?}

\section{A hedonic function estimate of dairy milk specific attributes.}

\begin{abstract}
In the context of a scheduled end of the milk quota system, the valuation of milk is a key variable in the profitability of dairy farms. Cow's milk derives its value from different products of its transformation. The origin and quality signs are a major differentiator for cheese specialties but also for butter and cream. Thus, the valuation of milk depends on its composition but also on qualitative attributes of the dairy product relating to its origin and its typicality. Based on the Farm Accounting Data Network (FADN), this work therefore aims to estimate the implicit value for the producer of the quantitative and qualitative attributes of the milk using a hedonic regression methodology applied to panel data in a period of strong change in the price of this commodity (2006-2008). On this basis, we give estimates of the implicit value of the fat and protein contents, as well as those of the various official and professional quality labels, taking into account the regional contexts studied, both in milk production and processing.
\end{abstract}

Keywords: milk quality, hedonic regression, quality labels, Farm Accountancy Data Network, France

Classification JEL : D2, Q11, Q12 


\section{Introduction}

Les évolutions récentes au niveau européen, qui se sont traduites par une variabilité accrue du prix du lait entre 2006 et 2009, plaident pour la mise en place rapide d'accords de contractualisation qui, en lissant les fluctuations du marché, permettraient d'une part de stabiliser les prix d'approvisionnement des opérateurs de la transformation laitière, et d'autre part de sécuriser le revenu des producteurs laitiers. En effet, dans les scenarii d'évolution du secteur laitier retenus pour l'horizon 2015, le prix du lait constitue une variable-clé susceptible d'orienter selon son niveau, l'ampleur et la fréquence de ses fluctuations vers des restructurations aux conséquences plus ou moins drastiques en termes de répartition territoriale et de démographie des producteurs laitiers, qu'ils soient spécialisés ou mixtes (Chatellier et al., 2013).

Le lait en tant que produit de base tire sa valeur des produits laitiers qui, hormis le lait frais, font l'objet d'une transformation plus ou moins élaborée : lait pasteurisé (écrémé, semi-écrémé, etc.), crème fraîche, glace, yaourt, beurre, fromage, lait en poudre et autres produits laitiers concentrés. Utilisés dans la fabrication de ces produits, les composants-clés du lait sont la Matière Grasse (MG), la Matière Protéique (MP) et l'eau. Puisque les produits laitiers mobilisent ces composants en proportions différentes, chacun d'entre eux chacun d'entre eux, hormis l'eau, présente une valeur présente une valeur dite «implicite ». Ces valeurs implicites peuvent être déduites soit du marché de détail des produits laitiers (Ladd et Suvannunt, 1976), soit du marché à la production où le lait est acheté par les transformateurs laitiers aux producteurs (Ladd et Martin, 1976). Si ces différents marchés transmettent efficacement les signaux de prix, alors les valeurs implicites dérivées des prix concédés aux éleveurs laitiers seront égales aux valeurs correspondantes dérivées du marché de détail (Gillmeister et al., 1996). Il s'agit donc de savoir si le système de commercialisation du lait transmet efficacement les prix du marché de détail - sur lequel se forment les prix des produits laitiers, au marché à la production - où se forment les transformateurs laitiers s'approvisionnent auprès des éleveurs.

Supposer que le prix incorpore également un signal de demande pour la qualité permet d'estimer les prix implicites des attributs qualitatifs du lait résultant de la perception des signes de qualité des produits laitiers. L'objectif de cet article est donc d'utiliser l'information recélée par les transactions commerciales pour estimer les valeurs implicites de l'ensemble des attributs du lait au niveau de l'exploitation agricole qu'ils soient quantitatifs ou qualitatifs, qu'ils portent sur des caractéristiques biophysiques comme sur des attributs de typicité ou d'origine, afin de pouvoir analyser l'efficacité de la transmission des prix par le système de commercialisation du lait. En se plaçant dans un cadre où producteurs et transformateurs de lait partagent un certain nombre d'informations sur les attributs du lait et où le prix du lait est une fonction déterminable à un aléa près de ces attributs, cet article présente une approche économétrique qui permet d'attribuer ex post une valeur 
aux attributs quantitatifs et qualitatifs du lait à partir de l'observation des transactions sur le marché du lait à la production.

Notre contribution se structure comme suit : dans une première partie, nous décrivons le système de rémunération des producteurs laitiers puis nous situons la place occupée par le système des signes de qualité au sein de la filière lait ; dans une seconde partie, nous présentons le dispositif d'enquête spécifique mis en place pour collecter les informations sur les attributs du lait; dans une troisième partie, nous proposons un cadre conceptuel, basé sur un modèle hédonique, pour analyser les niveaux de prix perçus par les producteurs de lait en France, puis nous précisons la spécification retenue à l'issue de tests adéquats ; la mise en œuvre de cette spécification hédonique permettant d'estimer les prix implicites des attributs tant qualitatifs que quantitatifs du lait, nous détaillons dans une quatrième partie les résultats obtenus. Enfin, nous discutons la pertinence de ces résultats pour conclure sur les implications en termes de politique laitière.

\section{Contexte productif de la filière laitière française}

\subsection{Le système de rémunération des producteurs de lait}

Pour le lait, historiquement, la composante rémunérée a été la matière grasse (base de fabrication du beurre et de la crème), les composantes protéiques se partageant le solde de la valeur du produit de base, générée par les produits transformés. Plus récemment, la répartition de la valeur s'est modifiée au profit des matières protéiques en raison de la modification des profils de consommation alimentaire dans les pays développés (effet de l'extension du mode de vie urbain, influence éventuelle de certaines prescriptions normatives comme les recommandations nutritionnelles concernant la consommation de graisse d'origine animale, etc.). Actuellement, le lait est rémunéré selon des caractéristiques biophysiques que sont les taux de matière grasse et de matière protéique, ainsi que selon certaines caractéristiques sanitaires. En France, depuis la Loi Godefroy (1969), le lait est payé selon des critères mesurables permettant une rémunération du produit en fonction de l'écart (positif ou négatif) aux normes de composition d'un "lait standard ", soit 38 grammes par litre $(\mathrm{g} / \mathrm{l})$ pour la matière grasse et $32 \mathrm{~g} / \mathrm{l}$ pour la matière protéique.

Depuis 1997 , la rémunération du producteur selon ces différents attributs du lait fait l'objet d'un accord de l'interprofession laitière (producteurs, coopératives et industriels privés) édictant des recommandations sous l'égide du Centre National Interprofessionnel de l'Economie Laitière (CNIEL). Ces recommandations, indexées sur les principaux marchés d'aval pour la filière, sont adaptées aux différents contextes régionaux dans le cadre de négociations interprofessionnelles spécifiques à chaque centre régional (CRIEL) pour aboutir à une grille de rémunération propre à chacune des zones définies par les 
CRIEL $^{1}$ où les différences régionales portent principalement pour la période de production étudiée sur les différentiels de matières grasse et protéique.

Depuis, cette pratique a été abandonnée sur injonction de la Direction générale de la concurrence, de la consommation et de la répression des fraudes, craignant d'enfreindre les règlements européens relatifs au droit de la concurrence du fait d'une fixation arbitraire du prix du lait (Emorine et Bailly, 2009). Dans cette période de transition, il est essentiel pour les autorités publiques de régulation de savoir si les systèmes en vigueur de rémunération de la filière laitière française retransmettent efficacement aux producteurs laitiers en termes de prix les préférences finales exprimées par les consommateurs.

Alors que la segmentation de marché des produits laitiers s'appuie de plus en plus sur une argumentation commerciale développée autour de leurs qualités, notamment leur typicité et leur origine, le lait à la production peut-il être considéré comme un produit de base relativement indifférencié, essentiellement caractérisé par ses caractéristiques biophysiques?

Face à cette interrogation, nous analysons les modalités du système de rémunération et leurs effets pour savoir si la valorisation du produit à la production peut s'étendre aux attributs symboliques d'origine, de typicité et de modalités de production du lait. Sous cette hypothèse, les prix payés aux producteurs laitiers par les transformateurs laitiers devraient être proportionnels à une somme pondérée des valeurs des composantes physiques et attributs qualitatifs du lait, pour ainsi tenir compte de l'ensemble des préférences des consommateurs perçues à travers les profils de consommation des produits laitiers, notamment par rapport aux signes de qualité dont ils sont porteurs.

\subsection{Les signes de qualité au sein de la filière laitière}

Les signes de qualité constituent un des facteurs majeurs de différenciation et de développement de l'offre agro-alimentaire (Lagrange et al., 2000). Ils mettent en exergue les spécificités des produits ayant trait à leur origine et aux conditions de leur élaboration, attestant du respect d'un cahier des charges visant à perpétrer certains procédés ou à protéger l'environnement.

L'évolution de l'offre alimentaire vers les produits transformés conduit à une diversification croissante des produits laitiers permise par l'innovation de procédés et de produits. Cette diversification est basée non seulement sur des attributs quantitatifs relevant de leur composition, mais également sur

\footnotetext{
${ }^{1}$ Jusqu'en mai 2008, le prix payé au producteur était indexé sur les prix constatés sur les marchés nationaux en aval pour les 12 mois précédents et sur les marchés mondiaux, la négociation s'appuyant sur le niveau des différents débouchés de la filière : produits de grande consommation (50\% du marché) et industriels sur le marché français, produits exportés.
} 
des attributs qualitatifs constitutifs de leur origine (Appellation d'Origine Contrôlée/Appellation d'Origine Protégée - AOC/AOP), de leur typicité (Indication Géographique Protégée - IGP), de leurs modes de production aux différents stades (Agriculture Biologique, Label Rouge, Certification de conformité de produit) ou de leur image véhiculée par des chartes (Charte des bonnes pratiques d'élevage), ou des marques (AgriConfiance). Ces signes de qualité du lait sont définis d'une part pour les signes officiels de qualité (AOC/AOP, Agriculture Biologique, IGP, Certification de conformité de produit) par l'Institut national de l'origine et de la qualité (INAO), d'autre part pour les signes professionnels par www.agriconfiance.coop pour la marque AgriConfiance, et par www.charte-elevage.fr pour la Charte des bonnes pratiques d'élevage.

Selon des analyses concordantes, les signes de qualité portant sur l'origine du produit jouent un rôle-clé dans la prise en compte des préoccupations des consommateurs concernant le lien entre alimentation et santé d'une part et, d'autre part, dans la réponse aux attentes sociales en matière d'environnement en favorisant les processus de dés-intensification de l'agriculture (Valceschini, 2008). Ils se révèlent être un des facteurs confortant le maintien des activités d'élevage et des petites exploitations, particulièrement dans les zones défavorisées de plateau, de piémont et de montagne (Aubert et Perrier-Cornet, 2009). Ainsi, le développement des signes de qualité constitue l'un des principaux leviers de l'intervention publique pour corriger les défaillances de marché entraînant des choix sous-optimaux pour le consommateur ou le producteur (Marette, 2009).

Les sources statistiques concernant la filière laitière française ${ }^{2}$ permettent de cerner l'importance économique des produits laitiers sous signes de qualité dont les spécialités fromagères d'appellation contrôlée au lait de vache constituent la part la plus emblématique. Sur la période étudiée (2006-2008), ces productions laitières sous AOC/AOP représentent un chiffre d'affaires d'1,5 milliards d'euros hors taxes et concernent entre 17 et $20 \%$ des producteurs français de lait de vache. En volume, la production de lait de vache sous AOC/AOP représente $9 \%$ de la collecte ; les fromages représentent $85 \%$ de sa transformation. En 2008, la part de marché des fromages AOC/AOP était de $21 \%$ en valeur.

Cependant, dans le moyen et le long terme, les goûts du consommateur ainsi que ses comportements d'achat peuvent évoluer significativement : en France entre 1997 et 2006, la consommation de produits laitiers a baissé de $7 \%$, principalement pour les laits conditionnés $(-20 \%)$ et le beurre $(-12 \%)$; par contre, si la consommation de fromages est stable, celle des yaourts augmente de moitié tandis que la part des desserts lactés est en forte croissance $(+70 \%)$.

${ }^{2}$ Cf. les sites de la statistique publique agricole (www.agreste.fr), de l'établissement public FranceAgriMer (www.franceagrimer.fr), du CNIEL (www.cniel.fr) et de l'INAO (www.inao.gouv.fr) pour les appellations d'origine des produits laitiers. 
Les changements que ces évolutions induisent dans la palette des produits offerts par les transformateurs laitiers affectent alors la valeur relative des attributs biophysiques et symboliques des produits transformés. Nous utilisons donc l'information de marché pour estimer à la production les valeurs implicites des attributs du lait utilisé comme intrant par l'industrie laitière et ainsi pouvoir analyser l'efficacité de la transmission des prix par le système de commercialisation du lait. Pour ce faire, nous spécifions la fonction de production du transformateur laitier selon le modèle des caractéristiques d'intrants (Ladd et Martin, 1976), en introduisant des attributs tant symboliques que biophysiques pour caractériser le lait comme facteur de production.

\section{Base empirique d'estimation : la Sonde laitière}

Le dispositif d'enquête spécifique mis en place pour collecter les informations sur les signes de qualité du lait est constitué par une enquête complémentaire au Réseau d'Information Comptable Agricole (RICA) dénommé "Sonde laitière ». La Sonde laitière est une enquête spécifique annexée au recueil de comptabilités du RICA géré par le Service de Statistique et de Prospective (SSP) du Ministère en charge de l'Agriculture pour collecter des informations sur la commercialisation du lait et les signes de qualité associés. Elle a été réalisée dans le cadre d'une convention avec le CNIEL. Mise en œuvre selon la méthodologie des quotas appliquée aux trois critères de stratification que sont la région, la Classe de Dimension Économique (CDEXE) et l'Orientation Technico-Économique (OTEXE), la sélection de l'échantillon RICA assure à la Sonde laitière une représentativité empirique vis-à-vis de la population des exploitations laitières dites «professionnelles ${ }^{3}$. Pour les enquêtes annuelles 2006-2007-2008 de la Sonde laitière, une exploitation agricole française est classée comme «professionnelle » si elle dépasse une taille économique minimale fixée à huit Unités de Dimension Économique (UDE), soit $9600 €$ (valeur 2004) de Marge Brute Standard (MBS, indicateur statistique de la marge brute réalisable). En France, la part des exploitations laitières «non-professionnelles » est marginale en effectif comme en dimension économique pour les OTEXE concernées : selon l'enquête «Structure des exploitations agricoles »d'Agreste, elles sont estimées en bovins-lait à deux mille exploitations et en bovins mixtes à trois cents exploitations, représentant au plus $1 \%$ de la marge brute de l'OTEXE considérée (Grivaux, 2008).

La cohorte retenue pour cette étude est ainsi définie à partir de la population des exploitations professionnelles productrices de lait de vache,

3 Selon la méthodologie du RICA européen (cf. http://ec.europa.eu/ agriculture/rica/methodology1_fr.cfm) à laquelle se conforme le RICA français, on entend par « exploitation agricole professionnelle » une exploitation suffisamment importante pour assurer à l'agriculteur une activité principale et un niveau de revenu lui permettant de faire vivre sa famille. 
enquêtées sur la période 2006-2008 et clôturant en année civile selon les règles instituées par le RICA. Les transactions entre producteurs et laiteries que représentent les livraisons de lait sont comptabilisées via un système de facturation géré par la laiterie sous forme de «fiches de paie » du lait livré par les producteurs, y compris les «ventes directes». Selon les instructions d'enquête de la Sonde laitière, "relèvent de la vente directe les quantités de lait quittant l'exploitation du producteur pour être vendues directement par celui-ci au consommateur, à des grossistes, à des commerçants pratiquant la vente au détail ou cédées gratuitement, sans l'intermédiaire d'une entreprise traitant ou transformant du lait».

Les signes de qualité sont déclarés par le producteur dans le cadre de la Sonde laitière si une fraction significative de sa production de lait est concernée, au moyen du référentiel suivant : AOC/AOP, IGP, Agriculture Biologique, Label Rouge, Certification de conformité de produit, Charte des bonnes pratiques d'élevage, et marque AgriConfiance.

Nous disposons d'une cohorte de 398 exploitations présentes sur les exercices comptables 2006-2007-2008 (panel cylindré) dont $86 \%$ de producteurs spécialisés (OTEXE bovins laits), le solde se partageant essentiellement entre les orientations laitières mixtes. Le nombre d'observations de la cohorte est conditionné par le nombre d'exploitations du RICA spécialisées en production de lait de vache (environ $19 \%$ des exploitations sur la période) ; d'autre part, le RICA étant un dispositif d'enquête volontaire, seulement $30 \%$ des producteurs de lait de vache de l'échantillon ont répondu au volet supplémentaire sur le lait de vache qui impliquait un dépouillement des facturations du produit sur l'année comptable; autre déterminant de la constitution de cette cohorte, le taux d'attrition global des exploitations du RICA d'une année sur l'autre demeure relativement modéré : il est estimé à $11 \%$ pour cette période.

La population représentée par cette cohorte est estimée à environ 22700 exploitations laitières, soit plus d'un tiers des producteurs spécialisés. Les caractéristiques de cette population sont résumées par les informations de cadrage suivantes :

- les six principales régions laitières (Rhône-Alpes, Bretagne, BasseNormandie, Pays de la Loire, Auvergne et Franche-Comté) représentent environ $80 \%$ de la population enquêtée ;

- les trois principales CDEXE y figurent au prorata approximatif de leur importance dans la production laitière ;

- selon le zonage CRIEL indiquant les délimitations des accords de prix régionaux, les fréquences zonales s'écartent des densités d'exploitation de la campagne 2006/2007 estimées par l'Office de l'élevage (Emorine et Bailly, 2009) mais restituent globalement la hiérarchie des principales zones de production. 
Compte tenu des informations relatives aux transactions entre les collecteurstransformateurs et les producteurs et à leur contexte productif, recueillies dans le cadre de la Sonde laitière, les déterminants du prix payé au producteur sont sélectionnés parmi, d'une part des covariants que sont les différentiels de matière grasse ou de matière protéique, et d'autre part des facteurs que sont les attributs qualitatifs qu'ils soient factuels (e.g., la localisation de l'exploitation) ou déclaratifs (les signes de qualité).

Les signes de qualité les plus fréquemment rencontrés sont, d'une part pour les signes professionnels, la Charte des bonnes pratiques d'élevage mise en œuvre par 33,3 \% des éleveurs enquêtés, et d'autre part pour les signes officiels, l'AOC/AOP. Les signes officiels sont majoritaires en Franche-Comté, Auvergne et Poitou-Charentes tandis que les signes professionnels prédominent en Bretagne, Haute-Normandie, Champagne-Ardenne, Lorraine, Alsace et Languedoc-Roussillon. Un nombre restreint d'exploitations peut varier sur la période dans leur déclaration des signes, cependant le test de Kruskal-Wallis basé sur la statistique des rangs montre que ces variations annuelles ne sont pas significatives, hormis pour le Label Rouge et la Certification de conformité de produit.

Afin de pouvoir caractériser le « mix-produit » de l'établissement laitier transformateur, identifié grâce aux éléments comptables de la facture, nous avons élaboré une typologie sur la base de l'Enquête annuelle laitière à partir du profil de production en produits laitiers établi selon la nomenclature européenne PRODCOM (Production commercialisée), convertis en équivalents litres de lait standard selon les normes fournies par la Fédération nationale des industries laitières. Cette caractérisation a été effectuée sur la base d'une classification automatique multicritère prenant en compte les pourcentages de lait incorporés dans chacun des produits : le résultat est une typologie en neuf classes ( Fromage AOC », «Fromage non AOC », «Beurre, laits, autres produits », " Produits frais », « Produits industriels », « Fromages frais », «Diversifié » «Collecteur », «non-différencié ») permettant de ventiler l'ensemble de ces établissements laitiers dans des catégories homogènes selon les pourcentages de produit de base incorporés dans les principales composantes de leur production.

\section{Méthodologie, spécification et estimation de la fonction de prix hédonique}

\subsection{La méthodologie des prix hédoniques et son application au secteur laitier}

La méthode des prix hédoniques est fondée sur le constat que certains biens ne sont pas homogènes et présentent des particularités susceptibles d'augmenter ou au contraire de diminuer le consentement à payer de l'éventuel acquéreur d'un bien porteur de la caractéristique recherchée, ici le transformateur 
laitier pour un attribut spécifique du lait, afin de pouvoir en bénéficier. Les considérations empiriques, initialement centrées sur les changements de spécification et les améliorations de la qualité, ont précédé la formalisation théorique démontrant le déplacement des courbes d'offre et de demande pour les caractéristiques étudiées (Rosen, 1974).

L'approche hédonique permet d'attribuer une valeur soit aux biens pour lesquels il n'y a pas ou pas encore de marché (biens environnementaux), soit aux caractéristiques spécifiques (éventuellement immatérielles) de certains produits permettant de les distinguer d'autres biens vendus sur le même marché. Elle procède en associant à chaque produit un vecteur de caractéristiques désignées comme les «attributs »du bien. Dans le secteur laitier, cette méthode a été utilisée pour attribuer des valeurs implicites aux composants physiologiques et aux scores de qualité bactériologique du lait à la production (Gillmeister et al., 1996). Plus récemment, elle a été utilisée pour mesurer le consentement à payer du consommateur des différents attributs d'un produit laitier (le camembert) dans le contexte de la grande distribution où les signes officiels de qualité se croisent avec les types de marques déclinés au plan national et/ou spécifiquement pour chaque enseigne (Hassan et Monier-Dilhan, 2002).

En transformation laitière, les fonctions de production sont souvent spécifiées selon une technologie de type Leontief (Liu et al., 1990), comme dans le modèle d'équilibre spatialisé de Zhu, Cox et Chavas (1999) et sa variante régionalisée à l'échelle européenne (Bouamra-Mechemache et al., 2002). Cependant, les technologies de transformation laitière se caractérisent par les propriétés suivantes : rendements d'échelle constants, courbes de production marginales inélastiques et faibles élasticités de substitution entre les différents composants du lait (Gillmeister et al., 1996).

Nous utilisons la spécification théorique du modèle d'allocation des caractéristiques d'intrants (Ladd et Martin, 1976) en l'étendant à des attributs qualitatifs et en l'appliquant à une entreprise industrielle du secteur de la transformation laitière confrontée au problème du choix optimum de ses intrants dans une optique de maximisation de ses profits. Selon (Gillmeister et al., 1996), la technologie de production pour cette entreprise de transformation laitière multi-produit peut s'écrire au moyen de la fonction de transformation implicite suivante :

$$
\mathrm{H}\left(Q_{1}, \ldots, Q_{N}, C_{1}, \ldots, C_{j}, C_{j+1}, \ldots, C_{j^{\prime}}, \ldots, C_{j^{\prime}}\right)=0
$$

sous les contraintes d'approvisionnement $C_{\mathrm{j}}=X_{\mathrm{j}} M$, et $C_{\mathrm{j}+\mathrm{k}}=Z_{\mathrm{k}} M$

avec $Q_{1}, \ldots, Q_{\mathrm{n}}$, les quantités de produits fournies,

$\left\{C_{1}, \ldots, C_{j}, \ldots, C_{J}\right\}$, les contraintes d'approvisionnement en caractéristiques quantitatifs des intrants, et $\left\{C_{J+1}, \ldots, C_{J+K}, \ldots, C_{J+K}\right\}$, les standards qualitatifs de la production, 
$X_{j}$, la teneur d'une unité de lait pour le $j^{e}$ attribut quantitatif, de lait,

$Z_{k}$, l'occurrence (présence/absence du ke attribut qualitatif pour une unité

\section{$M$, la quantité de lait achetée.}

En supposant que $H$ soit séparable pour les produits et non-jointe pour les intrants, l'équation (1) peut alors s'écrire sous la forme :

$$
\mathrm{H}=Q-F\left(C_{1}, \ldots, C_{j}, C_{J+1}, \ldots, C_{J+K}\right)=0
$$

où $Q=g\left(Q_{1}, \ldots, Q_{N}\right)$ est l'indice de production agrégé pour le secteur de la transformation laitière, laitière.

et $F$, une fonction de production agrégée du secteur de la transformation

Soit $p=p\left(p_{1}, \ldots, p_{h}, \ldots, p_{N}\right)$, l'indice agrégé du prix des produits laitiers, où $p_{h}$ est le prix du produit laitier $h$. Le problème d'optimisation peut alors s'écrire :

$$
\max \Pi=p F\left(C_{1}, \ldots, C_{j}, C_{J+1}, \ldots, C_{J+K}\right)-P_{M} M
$$

sous les contraintes d'approvisionnement $C_{j}=X_{j} M$, et $C_{j+k}=Z_{k} M$,

où $\Pi$ est le profit de l'entreprise de transformation laitière, et $P_{\mathrm{M}}$, le prix en euros du lait payé au producteur pour mille litres $(€ / \mathrm{Kl})$.

En faisant l'hypothèse que la fonction de production est homogène de degré 1, la condition du premier ordre pour la maximisation du profit s'écrit comme suit :

$$
\frac{\partial \pi}{\partial M}=\sum_{j=1}^{J} p \frac{\partial F}{\partial c_{j}} \frac{\partial c_{j}}{\partial M}+\sum_{k=1}^{K} p \frac{\partial F}{\delta c_{J+k}} \frac{\delta c_{J+k}}{\partial M}-P_{M}=0
$$

où $\frac{\partial c_{j}}{\partial M}=X_{j}$ est le rendement physique marginal du $j^{e}$ attribut quantitatif, et

$$
\frac{\Delta c_{j}+k}{\partial M}=Z_{k}
$$

le rendement marginal du $k^{e}$ attribut qualitatif, d'une unité de lait supplémentaire.

Le problème d'optimisation (3) a donc pour solution un prix optimal $P_{\mathrm{M}}$ *, défini par :

$$
P_{M}^{*}=\sum_{j=1}^{J} p \frac{\partial F}{\partial c_{j}} X_{j}+\sum_{k=1}^{K} p \frac{\partial F}{\delta c_{J+k}} Z_{k}
$$


Conformément aux caractéristiques des technologies de transformation laitière antérieurement évoquées, la valeur du produit marginal du $j^{\mathrm{e}}$ attribut quantitatif est donnée par un terme non-linéaire $u_{j}=p \frac{\partial F}{\partial c_{j}}$, où son produit physique marginal $\frac{\partial F}{\partial c_{j}}$ est valorisé par $p$, l'indice agrégé du prix des produits laitiers ; respectivement, la valeur $\mathrm{du}$ produit marginal $\mathrm{du} k^{\mathrm{e}}$ attribut qualitatif, est définie par le terme non linéaire $v_{k}=p \frac{\partial F}{\delta c_{J+k}}$. A l'équilibre de marché, ces valeurs peuvent être interprétées comme les prix marginaux implicites des attributs quantitatifs $j$ et des attributs qualitatifs $k$. L'équation (5) fournit ainsi une décomposition du prix optimum du lait payé aux producteurs selon les produits bruts marginaux en valeur des $J$ attributs quantitatifs, $u_{j} X_{j}=p \frac{\partial F}{\partial c_{j}} \times \frac{\partial c_{j}}{\partial M}$, auxquelles s'ajoutent ceux des produits bruts marginaux des $K$ attributs qualitatifs $v_{k} Z_{k}=p \frac{\partial F}{\delta c_{J+k}} \times \frac{\delta c_{J+k}}{\partial M}$, pour toute unité de lait supplémentaire.

Déterminé en (4) par la condition du premier ordre selon la formulation suivante :

$$
P_{M}^{*}=\sum_{j=1}^{J} u_{j} X_{j}+\sum_{k=1}^{K} v_{k} Z_{k}
$$

le prix optimum du lait $P_{M}$ * constitue l'expression analytique du modèle hédonique des prix du lait à la production.

Chaque établissement laitier étant supposé choisir un lait représentant un assortiment optimal d'attributs pour la maximisation de son profit, dans le respect de sa contrainte d'approvisionnement, le prix $P_{M}$ du lait à la production est déterminé par une fonction de prix hédonique $P_{M}(X, Z)$ où $X=\left\{X_{1}, \ldots, X_{j}, \ldots, X_{j}\right\}$ et $Z=\left\{Z_{1}, \ldots, Z_{k}, \ldots, Z_{K}\right\}$ sont des vecteurs dont chaque composante se définit comme suit : soit la quantité $X_{\mathrm{J}}(i)=X_{J}^{i}$ de la caractéristique $j$ mesurée ou dénombrée par quantum de lait de l'exploitation $i$, s'il s'agit d'un attribut quantitatif ;

soit la présence/absence $Z_{k}(i)=z_{k}^{i}=\left\{\begin{array}{l}1 \\ 0\end{array}\right.$

de la qualité $k$ pour le lait de l'exploitation $i$, s'il s'agit d'un attribut qualitatif.

Ainsi, la propension marginale à payer pour un changement dans la composition du lait est égale à la dérivée partielle $\frac{\partial P}{\partial x_{j}}$ de la fonction de prix hédonique relative à cet attribut. Pour les attributs qualitatifs, on admet que le taux d'accroissement $\frac{\Delta P}{\Delta z_{k}}$ représente la propension marginale à payer. Le prix implicite de l'attribut est donc défini selon son niveau de mesure par la dérivée partielle $\frac{\partial P}{\partial x_{j}}$ pour un attribut quantitatif ou bien par le taux d'accroissement $\frac{\Delta P}{\Delta z_{k}}$ pour un attribut qualitatif. 


\subsection{Modèle empirique et stratégie d'estimation}

Le cadre théorique d'équilibre concurrentiel (Rosen, 1974) au sein duquel est formalisé le concept de prix hédonique suggère que des formes fonctionnelles non-linéaires s'avèrent plus appropriées que des formes linéaires pour la fonction de prix hédonique (Ekeland et al., 2004). Nous choisissons une forme log-linéaire, la loi log-normale fournissant une distribution théorique bien adaptée aux prix intrinsèquement positifs. La spécification de cette fonction relève également de considérations empiriques (Halvorsen et Pollakowski, 1981). Ainsi, pour rendre compte de l'existence possible d'éléments non linéaires, la spécification de notre modèle comporte des termes polynomiaux du second degré en les indices des taux de matière grasse et de matière protéique ainsi que des termes d'interaction entre un certain nombre d'attributs qualitatifs (e.g., les signes de qualité n'étant pas a priori indépendants des autres attributs décrivant le contexte de production et de commercialisation).

Exprimée en données de panel, la spécification du modèle hédonique est testée à partir des hypothèses suivantes : i) les paramètres de pente sont homogènes selon les exploitations et constants au cours du temps ; ii) l'aléa comporte un effet spécifique à l'exploitation, un effet spécifique à la période d'observation, et un effet d'interaction indépendamment et identiquement distribué (iid) selon une loi normale de moyenne nulle et de variance constante. Compte tenu des résultats liés aux différents tests de spécification ${ }^{4}$, nous retenons un modèle à effets fixes temporels estimé par les moindres carrés ordinaires pondérés $(\mathrm{MCO})$ ) en périodes empilées. Aussi, pour capturer l'hétérogénéité individuelle, nous introduisons les caractéristiques suivantes de l'exploitation : appartenance à une zone CRIEL, signes de qualité du lait, mix-produit des établissements laitiers, statut coopératif du producteur et présence de vente directe.

Ainsi, les attributs quantitatifs et qualitatifs sélectionnés au terme de la procédure de spécification comme déterminants du prix du lait dans la modélisation hédonique sont les suivants : i) $X_{j}=\frac{Q_{j}}{Q_{j}^{0}}$, indices de taux de matière grasse $(\mathrm{MG}, j=1)$ et de taux de matière protéique $(\mathrm{MP}, j=2)$ calculés par rapport aux normes de composition du lait standard $Q_{M G}^{0}=38 \mathrm{~g} / \mathrm{l}$; $Q_{M P}^{0}=32 \mathrm{~g} / \mathrm{l}$ - les données sont extraites des facturations comptables mensuelles établies par l'établissement laitier collecteur et/ou transformateur ; ii) $l_{\mathrm{t}}$, l'année civile codée en variable dichotomique pour capturer l'effet propre des variations annuelles en prix constants ; iii) $Z_{\text {d, }}^{\prime}$, variables dichotomiques codant l'appartenance à une zone CRIEL où s'applique une grille spécifique de rémunération déterminée d'après la géo-localisation du siège de l'exploitation agricole et codée sous forme qualitative ; iv) $Z_{e}$, variables dichotomiques

${ }^{4}$ Rejet de l'hypothèse des effets aléatoires (test de Hausman) et effets fixes individuels non significatifs; les résultats détaillés des tests sont disponibles auprès de l'auteur. 
renseignant sur l'attribution au lait des signes de qualités tant officiels que contractuels ; elle constitue une information déclarative, fournie par le producteur laitier) $Z_{f}^{\prime}$, variable dichotomique caractérisant le mix-produit des établissements laitiers collecteurs et/ou transformateurs ; la catégorisation utilisée est élaborée sur la base de l'Enquête annuelle laitière selon le profil de production en produits laitiers (convertis en équivalents litres de lait standard selon les normes de l'industrie laitière) ; $Z_{g}^{\prime}$, variable dichotomique indiquant le statut de coopérateur ou non du producteur laitier ; $Z^{\prime}{ }_{h}$, variable dichotomique codant la présence de ventes directes de lait effectuées auprès de clients non professionnels de la collecte ou de la transformation laitière.

Les moyennes des attributs quantitatifs et les fréquences des attributs qualitatifs au sein de la cohorte, avec les écarts-types correspondants, figurent au tableau 1 ainsi que les minima et maxima observés.

Les prix du lait (cf. Tableau 1) sont exprimés en euros constants, obtenus après déflation par l'indice des prix des produits laitiers de l'industrie pour le marché français (CPF 10.5, base 2010, INSEE). Les tests non-paramétriques réalisés pour les trois échantillons annuels appariés confirment des différences inter-annuelles de prix significatives pour la période étudiée : les prix réels sont les plus élevés en 2008 (en moyenne, 375,87 $€ / \mathrm{Kl}$ ), suivis par les prix 2007 $(312,86 €)$ tandis que les prix 2006 les plus faibles $(286,94 € / \mathrm{Kl})$; l'analyse de la variance montre que la variabilité inter-annuelle représente $43 \%$ de la variabilité totale des prix ; le coefficient de variation met en évidence une décroissance de cette variabilité inter-annuelle en 2007, puis en 2008.

Selon les mêmes statistiques de test, les différences inter-annuelles sur les taux de matière grasse et de matière protéique ne sont pas significatives : les taux de matière grasse et de matière protéique restent stables sur la période étudiée, autour de 41,54 grammes de matière grasse par litre de lait (41,54 g/l) et de 33,36 g/l de matière protéique. Les changements de profils en attributs qualitatifs au cours de la période sont peu nombreux (entre 1,5\% et 6,5\% des exploitations) et, hormis pour le Label Rouge et la Certification de conformité de produit, non significatifs d'après les tests d'indépendance réalisés.

Dans le cadre de cette modélisation, le prix de référence $P$ est défini par rapport à un "lait standard " en termes de taux de matière grasse (38 g/l) et de matière protéique $(32 \mathrm{~g} / \mathrm{l})$, collecté dans l'année 2006 auprès de producteurs de Savoie rémunérés selon les règles de l'accord interprofessionnel laitier, sans les signes distinctifs de qualité sélectionnés, vendu pour la production de spécialités laitières non spécifiques à des transformateurs dont le mix-produit apparaît comme indifférencié, qualités qui forment les catégories de référence. L'estimation du prix de référence obtenue à partir de notre échantillon est égale à $404,86 €$ pour mille litres de lait $(€ / \mathrm{Kl})$, ciblant ainsi une rémunération supérieure à celle obtenue en moyenne pour les laits commercialisés par notre échantillon d'exploitations. 
Tableau 1 : Caractérisation des variables du modèle empirique.

\begin{tabular}{|c|c|c|c|c|c|}
\hline \multicolumn{2}{|l|}{$\begin{array}{l}\text { Attributs } \\
\text { quantitatifs }\end{array}$} & Moyenne & Écart-type & Minimum & Maximum \\
\hline \multicolumn{2}{|l|}{ Prix du lait $(€ / K l)$} & 324,26 & 354,14 & 236,19 & 554,55 \\
\hline \multicolumn{2}{|l|}{$\begin{array}{l}\text { Taux de matière grasse } \\
(\mathrm{g} / \mathrm{l})\end{array}$} & 41,54 & 20,06 & 31,69 & 56,18 \\
\hline \multicolumn{2}{|l|}{$\begin{array}{l}\text { Taux de matière } \\
\text { protéique }(g / l)\end{array}$} & 33,36 & 10,61 & 29,08 & 44,92 \\
\hline \multicolumn{6}{|l|}{$\begin{array}{l}\text { Attributs } \\
\text { qualitatifs } \\
\text { Zones CRIEL }\end{array}$} \\
\hline \multirow{4}{*}{$\begin{array}{l}\text { Catégorie de référence } \\
\text { Autres catégories }\end{array}$} & Savoie & 0,0642 & 1,6873 & 0 & 1 \\
\hline & Autres Zones CRIEL & 0,8194 & 2,7317 & 0 & 1 \\
\hline & Hors Zones CRIEL & 0,1061 & 2,1871 & 0 & 1 \\
\hline & Haute-Normandie & 0,0103 & 0,7163 & 0 & 1 \\
\hline \multicolumn{6}{|l|}{ Signe de qualité } \\
\hline Catégorie de référence & $\begin{array}{l}\text { Sans signe ou autres } \\
\text { signes }\end{array}$ & 0,8022 & 2,8289 & 0 & 1 \\
\hline \multirow[t]{4}{*}{ Autres catégories } & IGP & 0,0396 & 1,3856 & 0 & 1 \\
\hline & Label Rouge & 0,0166 & 0,9069 & 0 & 1 \\
\hline & AOC/AOP & 0,1426 & 2,4829 & 0 & 1 \\
\hline & $\begin{array}{l}\text { Agriculture } \\
\text { Biologique }\end{array}$ & 0,0212 & 1,0228 & 0 & 1 \\
\hline \multicolumn{6}{|l|}{ Mix-produit } \\
\hline Catégorie de référence & Non différencié & 0,9066 & 2,0669 & 0 & 1 \\
\hline Autre catégorie & $\begin{array}{l}\text { Fromages et } \\
\text { produits frais }\end{array}$ & 0,0934 & 2,0669 & 0 & 1 \\
\hline \multicolumn{6}{|l|}{ Statut } \\
\hline Catégorie de référence & Non-coopérateur & 0,3246 & 3,3252 & 0 & 1 \\
\hline \multicolumn{6}{|l|}{ Commercialisation } \\
\hline Catégorie de référence & Sans vente directe & 0,9617 & 1,3631 & 0 & 1 \\
\hline Autre catégorie & Vente directe & 0,0383 & 1,3631 & 0 & 1 \\
\hline
\end{tabular}

Source : RICA 2006-2008, Sonde laitière, calculs de l'auteur.

\section{Les résultats économétriques de la modélisation empirique}

Compte tenu de ce qui précède, nous estimons la forme log-linéaire suivante correspondant à un modèle empirique à effets temporels fixes :

$$
\begin{aligned}
\ln \left(p_{M(i, t)}\right)= & \alpha_{0}+\sum_{j=1}^{J} \beta_{j} x_{i t}^{j}+\sum_{j=1}^{J} \beta_{j j^{\prime}}\left(x_{i t}^{j}\right)^{2}+\alpha_{t} I_{t}+\sum_{k=1}^{K} \delta_{k} \bar{Z}_{i}^{k} \\
& +\sum_{k=1}^{K} \sum_{l=1}^{L} \delta_{k l} \bar{Z}_{i}^{k} \bar{Z}_{i}^{l}+\varepsilon_{i t}^{\prime}
\end{aligned}
$$


où $P_{\mathrm{M}(\mathrm{i}, \mathrm{t})}$ constitue le prix du lait payé à l'exploitation $i$ par l'établissement laitier pour la période $t ; x_{i t}^{j} i=1, \ldots, I ; j=1, \ldots, J$ est la valeur de l'attribut quantitatif $j$ pour l'exploitation $i$ au cours de l'année $t$; $l_{\mathrm{t}}, t=1, \ldots, T-1$ sont les indicatrices de codage des années de la période d'observation $T ; \quad \bar{Z}_{i}^{k} k=1, \ldots, K$ sont les indicatrices de codage des attributs qualitatifs, supposés constants au cours de la période ; $\epsilon^{\prime}{ }_{i t}$ est un aléa iid selon une loi normale $N(0, \sigma)$.

Dans une première section sont présentées la validation et les estimations du modèle empirique (équation 7) obtenues à la situation de référence - définie par la composition du lait standard $\left(\mathrm{Q}_{\mathrm{MG}}^{0}=38 \mathrm{~g} / \mathrm{I} ; \mathrm{Q}_{\mathrm{MP}}^{0}=32 \mathrm{~g} / \mathrm{I}\right)$ et les catégories de référence - en termes de paramètre du modèle, d'élasticité partielle $e^{5}$ pour les attributs quantitatifs correspondant aux teneurs en matières grasses et protéiques, d'effet discret ${ }^{6}$ pour les attributs qualitatifs selon la préconisation de Kennedy (1981), ainsi que les prix implicites en résultant ; dans une seconde section, ces estimations sont interprétées par rapport aux enjeux économiques de la filière lait en France, en particulier ceux liés à la valorisation des signes de qualité.

\subsection{Validation de la spécification et estimations du modèle empirique}

La spécification du modèle hédonique est validée par les tests suivants : i) l'hypothèse d'indépendance entre les régresseurs et les résidus n'est pas rejetée par le test d'Hausman ; ii) l'hypothèse de normalité des résidus est rejetée par le test de Kolmogorov-Smirnov, en raison d'une distribution leptokurtique et d'une faible asymétrie par valeurs supérieures ; iii) l'hypothèse d'homoscédasticité est rejetée par le test de White (1980). L'estimation est donc effectuée selon une procédure des moindres carrés pondérés par l'inverse du taux d'échantillonnage, ce qui permet d'obtenir des estimations valides pour la population échantillonnée mais a également pour effet de limiter l'hétéroscédasticité.

La matrice hessienne dérivée de cette spécification est définie négative compte tenu des valeurs estimées de ces paramètres ( $c f$. Tableau 2), vérifiant

${ }^{5}$ Les élasticités sont calculées pour $Q_{j}, j=M P, M G$ comme suit : $\varepsilon\left(P, Q_{j}\right)=\frac{\partial P}{\partial Q_{j}} \times$ $\frac{Q_{j}}{P}$, soit $\varepsilon_{\mathrm{j}}\left(\mathrm{P}^{\circ}, \mathrm{Q}^{\circ}\right)=\left(\hat{\beta}_{\mathrm{j}}+\hat{\beta}_{\mathrm{jj}}\right)$.

${ }^{6}$ L'effet discret introduit par un attribut qualitatif du lait se définit comme la variation relative du prix à la production lorsque la variable binaire indicatrice de cet attribut passe de la valeur zéro (codant l'absence de l'attribut) à la valeur un (codant sa présence). L'effet discret de l'attribut qualitatif $m$ est calculé comme suit :
$\lambda\left(P, Q_{m}\right)=\frac{\Delta P}{\Delta Q_{m}} \times \frac{1}{P}$, soit
$\lambda_{\mathrm{m}}\left(\mathrm{P}^{\circ}, \mathrm{Z}^{\circ}\right)=\exp \left(\hat{\beta}_{\mathrm{m}}+\frac{1}{2} \hat{\mathrm{V}}\left[\hat{\beta}_{\mathrm{m}}\right]\right)-$

1 avec $\hat{\beta}_{\mathrm{m}}=\hat{\alpha}_{\mathrm{t}}, \hat{\delta}_{\mathrm{k}}$ ou $\hat{\delta}_{\mathrm{kl}}$. 
ainsi les conditions suffisantes du second ordre pour la concavité de notre fonction de prix en les taux de matière grasse et de matière protéique.

Les résultats de l'estimation ( $c f$. Tableau 2) sont obtenus d'après un modèle optimisé dont les variables explicatives sont sélectionnées suivant la règle du $C_{p}$ de Mallows ${ }^{7}$ (1973). Bien que toutes les interactions entre attributs qualitatifs ne soient pas estimables en raison de colinéarités induites, certaines interactions, comme celles entre l'appartenance aux zones CRIEL et les signes de qualité ou le statut coopératif du producteur laitier, ainsi que celle entre certains signes de qualité (IGP) et le mix-produit, sont significatives selon le test du F de Fisher ${ }^{8}$ opposant le modèle sans interaction au modèle avec interactions. Cette dernière spécification présente un excellent pourcentage $(91,2 \%)$ de variabilité expliquée des prix du lait à la production. Les résultats du test RESET confirment la spécification fonctionnelle adoptée.

L'estimation des effets marginaux pour l'ensemble des variables sélectionnées ( $c f$. Tableau 3) s'effectue relativement au prix de référence $P^{\mathrm{O}}$, égal à 404,86 $€$ pour mille litres de lait $(€ / \mathrm{Kl})$, (cf. section 3.2) pour un lait standard collecté en 2006 auprès des producteurs savoyards, sans signe de qualité ni mix-produit particuliers.

Les prix 2006 étant les plus bas de la période retenue, les effets annuels marginaux sur le prix du lait sont positifs, soit $37,15 € / \mathrm{Kl}$ pour l'année 2007 et $149,14 € / \mathrm{Kl}$ pour l'année 2008. Compte tenu de la déflation opérée avec l'indice du prix des produits laitiers, ces estimations s'interprètent comme les variations annuelles propres aux transactions analysées; elles sont cohérentes avec le niveau et l'évolution globale des prix à la production au cours des campagnes laitières concernées.

\subsubsection{Analyse des attributs quantitatifs du lait}

En ce qui concerne les différentiels de matière grasse et de matière protéique, la valeur implicite attribuable au différentiel unitaire de matière protéique par litre de lait $(+1 \mathrm{~g} / \mathrm{l}$ en MP) est de $10,10 €$ pour 1000 litres $(€ / \mathrm{Kl})$ de lait, correspondant à une élasticité estimée à 0,7982 . Prenant en compte le coefficient linéaire et le coefficient quadratique, ce prix implicite dépasse celui du différentiel unitaire de matière grasse, dont le niveau est de $4,48 € / \mathrm{Kl}$ correspondant à une élasticité estimée à 0,4203 . À la situation de référence, les simulations effectuées à partir des performances des races laitières alpines démontrent localement une meilleure valorisation marginale de la matière

${ }^{7}$ La statistique se calcule comme suit : $C_{q}=\frac{S C E_{p}}{s^{2}}-(n-2 q)$ avec $s^{2}$, le carré moyen des erreurs du modèle complet ; $q$, le nombre de paramètres, $S C E_{\mathrm{q}}$, la somme des carrés des erreurs du modèle sélectionné ; et $n$, le nombre d'observations.

${ }^{8}$ La valeur estimée du rapport $\mathrm{F}$ à 4 et 1173 degrés de libertés, de la variance des interactions à celle des erreurs du modèle complet, conduit à un risque de première espèce inférieur à $10^{-13}$. 
Tableau 2 : Résultats économétriques du modèle empirique.

\begin{tabular}{|c|c|c|c|}
\hline Déterminants & Covariants & Coeff & ficient \\
\hline Attributs quantitatifs & $\begin{array}{l}\text { Index MG } \\
(\text { Index MG) } \\
\text { Index MP } \\
(\text { Index MP) }\end{array}$ & $\begin{array}{c}\text { Valeur } \\
1,425 * * * \\
-1,005 * * * \\
2,991 * * * \\
-2,193 * * *\end{array}$ & $\begin{array}{c}t \text { de Student } \\
4,66 \\
-3,67 \\
5,01 \\
-3,96\end{array}$ \\
\hline $\begin{array}{l}\text { Attributs qualitatifs } \\
\text { Année, cat. ref. }\end{array}$ & $\begin{array}{l}\text { Catégories } \\
\text { Année } 2006 \\
\text { Année } 2007 \\
\text { Année } 2008\end{array}$ & $\begin{array}{l}0,088 * * * \\
0,314 * * *\end{array}$ & $\begin{array}{l}25,43 \\
91,84\end{array}$ \\
\hline Zones CRIEL, cat. ref. & $\begin{array}{c}\text { Savoie } \\
\text { Autres Zones CRIEL } \\
\text { Hors Zones CRIEL } \\
\text { Haute-Normandie }\end{array}$ & $\begin{array}{l}-0,389 * * * \\
-0,396 * * * \\
-0,327 * * *\end{array}$ & $\begin{array}{l}-17,46 \\
-21,58 \\
-15,81\end{array}$ \\
\hline $\begin{array}{l}\text { Signe de qualité, cat. ref. } \\
\text { ، }\end{array}$ & $\begin{array}{c}\text { Sans signe ou autres signes } \\
\text { IGP } \\
\text { Label Rouge } \\
\text { AOC/AOP } \\
\text { Agriculture Biologique }\end{array}$ & $\begin{array}{c}-0,070 * * * \\
0,024 * \\
0,069 * * * \\
0,185 * * *\end{array}$ & $\begin{array}{c}-7,07 \\
1,91 \\
11,33 \\
17,49\end{array}$ \\
\hline $\begin{array}{l}\text { Mix-produit, cat. ref. } \\
\text { Statut, cat. ref. }\end{array}$ & $\begin{array}{l}\text { Non différencié } \\
\text { Fromages et produits frais } \\
\text { Non-coopérateur }\end{array}$ & $0,027 * * *$ & 4,82 \\
\hline Commercialisation, cat. ref. & $\begin{array}{l}\text { Coopérateur } \\
\text { Sans vente directe }\end{array}$ & $-0,134 * * *$ & $-7,12$ \\
\hline & $\begin{array}{l}\text { Vente directe } \\
\text { Interactions }\end{array}$ & $0,017 * *$ & 2,10 \\
\hline $\begin{array}{l}\text { Zones CRIEL } \times \text { Signe de } \\
\text { qualité }\end{array}$ & Haute-Normandie $\times$ AOC/AOP & $-0,144 * * *$ & $-4,35$ \\
\hline Zone CRIEL $\times$ Statut & $\begin{array}{c}\text { Hors Zones CRIEL } \times \\
\text { Coopérateur }\end{array}$ & $0,096 * * *$ & 4,45 \\
\hline Zone CRIEL $\times$ Statut & $\begin{array}{c}\text { Autres Zones CRIEL } \times \\
\text { Coopérateur }\end{array}$ & $0,127 * * *$ & 6,65 \\
\hline $\begin{array}{l}\text { Signe de qualité } \times \text { Mix-Produit } \\
\mathrm{R}^{2} \text { ajusté }\end{array}$ & $\begin{array}{c}\text { IGP } \times \text { Fromages et produits frais } \\
\text { Constante }\end{array}$ & $\begin{array}{l}0,054 * * \\
0,884 * * * \\
0,912\end{array}$ & $\begin{array}{l}2,42 \\
2,82\end{array}$ \\
\hline
\end{tabular}

Source : RICA 2006-2008, Sonde laitière, calculs de l'auteur.

Lecture du tableau :

- les modalités en italique codent les catégories de référence (cat. ref.) correspondant à la situation du prix de référence ;

- le degré de significativité statistique des coefficients est exprimé selon les niveaux suivants du risque de première espèce : $\left(^{*}\right)$ inférieur à 10 pour cent ; $\left(^{* *}\right)$ inférieur à 5 pour cent ; $\left(^{* *}\right)$ inférieur à 1 pour cent.

protéique par rapport à la matière grasse : soit respectivement $8,92 € / \mathrm{Kl}$ contre 4,38 $€ / \mathrm{Kl}$ pour l'Abondance et $9,32 € / \mathrm{Kl}$ contre 4,64 $€ / \mathrm{Kl}$ pour la Tarentaise. Des estimations effectuées par rapport à d'autres situations de référence permettraient de tester d'éventuelles extensions ou modulations de cette conclusion. 
Tableau 3 : Estimation des élasticités, effets discrets et prix implicites au prix de référence $\mathrm{P}^{\circ}$.

\begin{tabular}{|c|c|c|c|c|c|}
\hline \multirow[b]{2}{*}{ Attributs } & & \multirow{2}{*}{$\begin{array}{l}\text { Élasticité } \\
\text { partielle }\end{array}$} & \multicolumn{3}{|c|}{ Prix implicite } \\
\hline & & & \multirow{2}{*}{$\begin{array}{c}\begin{array}{c}\text { Valeur } \\
(\boldsymbol{€} / \mathbf{K} \mathbf{l})\end{array} \\
(1)\end{array}$} & \multicolumn{2}{|c|}{$\begin{array}{l}\text { Intervalle de confiance à } \\
95 \%\end{array}$} \\
\hline \multirow{3}{*}{$\begin{array}{l}\text { Attributs } \\
\text { quantitatifs }\end{array}$} & Covariants & & & $\begin{array}{l}\text { Borne } \\
\text { inférieure }\end{array}$ & $\begin{array}{l}\text { Borne } \\
\text { supérieure }\end{array}$ \\
\hline & $\begin{array}{l}\text { Matière grasse } \\
(\mathrm{g} / \mathrm{l})\end{array}$ & 0,4203 & 4,48 & 4,44 & 4,52 \\
\hline & $\begin{array}{l}\text { Matière } \\
\text { protéique } \\
(\mathrm{g} / \mathrm{l})\end{array}$ & 0,7982 & 10,10 & 10,03 & 10,17 \\
\hline \multirow{4}{*}{$\begin{array}{l}\text { Attributs } \\
\text { qualitatifs } \\
\text { Année, cat. ref. }\end{array}$} & Catégories & Effet discret & (2) & & \\
\hline & Année 2006 & & & & \\
\hline & Année 2007 & 0,0918 & 37,15 & 34,41 & 39,88 \\
\hline & Année 2008 & 0,3684 & 149,14 & 146,44 & 151,85 \\
\hline \multirow{4}{*}{$\begin{array}{l}\text { Zones CRIEL, } \\
\text { cat. ref. }\end{array}$} & Savoie & & & & \\
\hline & $\begin{array}{l}\text { Haute- } \\
\text { Normandie }\end{array}$ & $-0,3274$ & $-132,55$ & $-150,25$ & $-114,85$ \\
\hline & $\begin{array}{l}\text { Autres Zones } \\
\text { CRIEL }\end{array}$ & $-0,3228$ & $-130,67$ & $-145,25$ & $-116,10$ \\
\hline & $\begin{array}{l}\text { Hors Zones } \\
\text { CRIEL }\end{array}$ & $-0,2790$ & $-112,97$ & $-129,38$ & $-96,56$ \\
\hline \multirow[t]{5}{*}{$\begin{array}{l}\text { Signe de qualité, } \\
\text { cat. ref. }\end{array}$} & $\begin{array}{l}\text { Sans signe ou } \\
\text { autres signes }\end{array}$ & & & & \\
\hline & IGP & $-0,0676$ & $-27,39$ & $-35,25$ & $-19,52$ \\
\hline & Label Rouge & 0,0246 & 9,95 & $-0,15$ & 20,06 \\
\hline & $\mathrm{AOC} / \mathrm{AOP}$ & 0,0717 & 29,02 & 24,17 & 33,87 \\
\hline & $\begin{array}{l}\text { Agriculture } \\
\text { Biologique }\end{array}$ & 0,2033 & 82,29 & 73,90 & 90,69 \\
\hline \multirow{2}{*}{$\begin{array}{l}\text { Mix-produit, } \\
\text { cat. ref. }\end{array}$} & Non différencié & & & & \\
\hline & $\begin{array}{l}\text { Fromages et } \\
\text { produits frais }\end{array}$ & 0,0276 & 11,19 & 6,69 & 15,68 \\
\hline \multirow{3}{*}{$\begin{array}{l}\text { Statut, cat.ref. } \\
\text { Commercialisation, } \\
\text { cat. ref. }\end{array}$} & Non-coopérateur & & & & \\
\hline & $\begin{array}{l}\text { Coopérateur } \\
\text { Sans vente directe }\end{array}$ & $-0,1255$ & $-50,81$ & $-65,73$ & $-35,88$ \\
\hline & $\begin{array}{l}\text { Vente directe } \\
\text { Interactions }\end{array}$ & 0,0171 & 6,91 & 0,50 & 13,32 \\
\hline $\begin{array}{l}\text { Zones CRIEL* } \\
\text { Signe de qualité }\end{array}$ & $\begin{array}{l}\text { Haute- } \\
\text { Normandie } * \\
\text { AOC/AOP }\end{array}$ & $-0,1350$ & $-54,65$ & $-81,01$ & $-28,30$ \\
\hline
\end{tabular}




\begin{tabular}{|c|c|c|c|c|c|}
\hline $\begin{array}{l}\text { Zone CRIEL * } \\
\text { Statut }\end{array}$ & $\begin{array}{l}\text { Hors Zones } \\
\text { CRIEL * } \\
\text { Coopérateur }\end{array}$ & 0,1007 & 40,77 & 23,62 & 57,93 \\
\hline $\begin{array}{l}\text { Zone CRIEL * } \\
\text { Statut }\end{array}$ & $\begin{array}{l}\text { Autres Zones } \\
\text { CRIEL * }\end{array}$ & 0,1354 & 54,84 & 39,66 & 70,02 \\
\hline $\begin{array}{l}\text { Signe de qualité* } \\
\text { Mix-Produit }\end{array}$ & $\begin{array}{l}\text { Coopérateur } \\
\text { IGP * } \\
\text { Fromages et } \\
\text { produits frais }\end{array}$ & 0,0554 & 22,43 & 4,69 & 40,18 \\
\hline
\end{tabular}

Source : RICA 2006-2008, Sonde laitière, calculs de l'auteur.

Lecture du tableau : les modalités en italique codent les attributs correspondant à la situation du prix de référence ; pour un prix de référence $\mathrm{P}^{\circ}$ de $404,86 € / \mathrm{Kl}$ et par rapport à des teneurs standards en matière grasse $\mathrm{Q}^{\circ}{ }_{\mathrm{MG}}=38 \mathrm{~g} / \mathrm{l}$ et en matière protéique $\mathrm{Q}^{\circ} \mathrm{MP}=32 \mathrm{~g} / \mathrm{l}$

(1) le prix implicite, $\Delta \mathrm{P}^{\circ}$, est calculé pour une variation unitaire $(=1 \mathrm{~g} / \mathrm{l})$ de la teneur $\mathrm{Qj}$ en l'attribut quantitatif $j$, soit matière grasse, soit matière protéique ; par ex., le prix implicite d'un gramme supplémentaire de matière protéique supplémentaire par litre de lait est de $10,10 € / \mathrm{Kl}$, avec $95 \%$ de chance d'être compris dans l'intervalle de prix $[10,03 ; 10,17]$.

(2) le prix implicite, $\Delta \mathrm{P}^{\circ} \mathrm{m}$, est calculé en différentiel de la présence $\left(\Delta \mathrm{Z}_{\mathrm{m}}=1\right)$ par rapport à l'absence $\left(\mathrm{Z}^{\circ} \mathrm{m}\right)$, catégorie de référence, pour l'attribut qualitatif $\mathrm{m}$; par ex., le prix implicite du signe de qualité Agriculture Biologique est de $82,29 € / \mathrm{Kl}$ avec $95 \%$ de chance d'être compris dans l'intervalle de prix $[73,90 ; 90,69]$.

Une simulation ${ }^{9}$ ( $c f$. Figure 1) dans le contexte du prix de référence $\mathrm{P}^{\circ}$ (404,86 $€ / \mathrm{Kl}$ en 2006) illustre la réponse en termes de prix aux variations des taux de matière grasse et de matière protéique. La courbe marginale du prix implicite de la matière grasse donne l'optimum partiel à 53,89 $\mathrm{g} / \mathrm{l}$ et celle du prix implicite de la matière protéique, l'optimum partiel à 43,65 g/l. Compte tenu de la précision obtenue pour le calcul de ces optima, les intervalles d'estimation sont proches des limites supérieures des intervalles de variation empirique de l'échantillon, soit pour la matière grasse $[31,69 ; 56,18] \mathrm{g} / \mathrm{l}$, et pour la matière protéique $[29,08 ; 44,92] \mathrm{g} / \mathrm{l}$. Selon l'interpolation de la fonction quadratique, l'optimum conjoint pour les prix $\mathrm{P}^{*}$ serait de $511,19 €$ pour 1000 litres de lait, en 2006.

Au-delà des optima partiels, les effets marginaux sur le prix décroissent plus rapidement pour la matière protéique que pour la matière grasse ; si bien qu'en rapport constant MG/MP correspondant au lait standard, ces effets s'égalisent pour $\mathrm{MG}=50,09 \mathrm{~g} / \mathrm{l}$ et $\mathrm{MP}=42,18 \mathrm{~g} / \mathrm{l}$. Eu égard au net ralentissement du progrès génétique laitier observé par l'Institut de l'élevage (Idele, 2014) depuis les années 2000, les prévisions actuelles sur les taux butyreux et protéique pour la période 2013-2019 amènent à

${ }^{9}$ Par dérivation partielle de la forme fonctionnelle, on obtient une estimation du gradient de la fonction de prix hédonique fournissant une approximation du prix implicite marginal pour les index de matière grasse $(I M G, j=1)$ et de matière protéique (IMP, $j=2$ ). Les optima correspondants sont obtenus en appliquant la condition du premier ordre à la forme fonctionnelle estimée. 
Figure 1: Distribution du prix du lait en fonction des composants protéique (MP) et lipidique (MG). Source : RICA 2006-2008, Sonde laitière, calculs de l'auteur

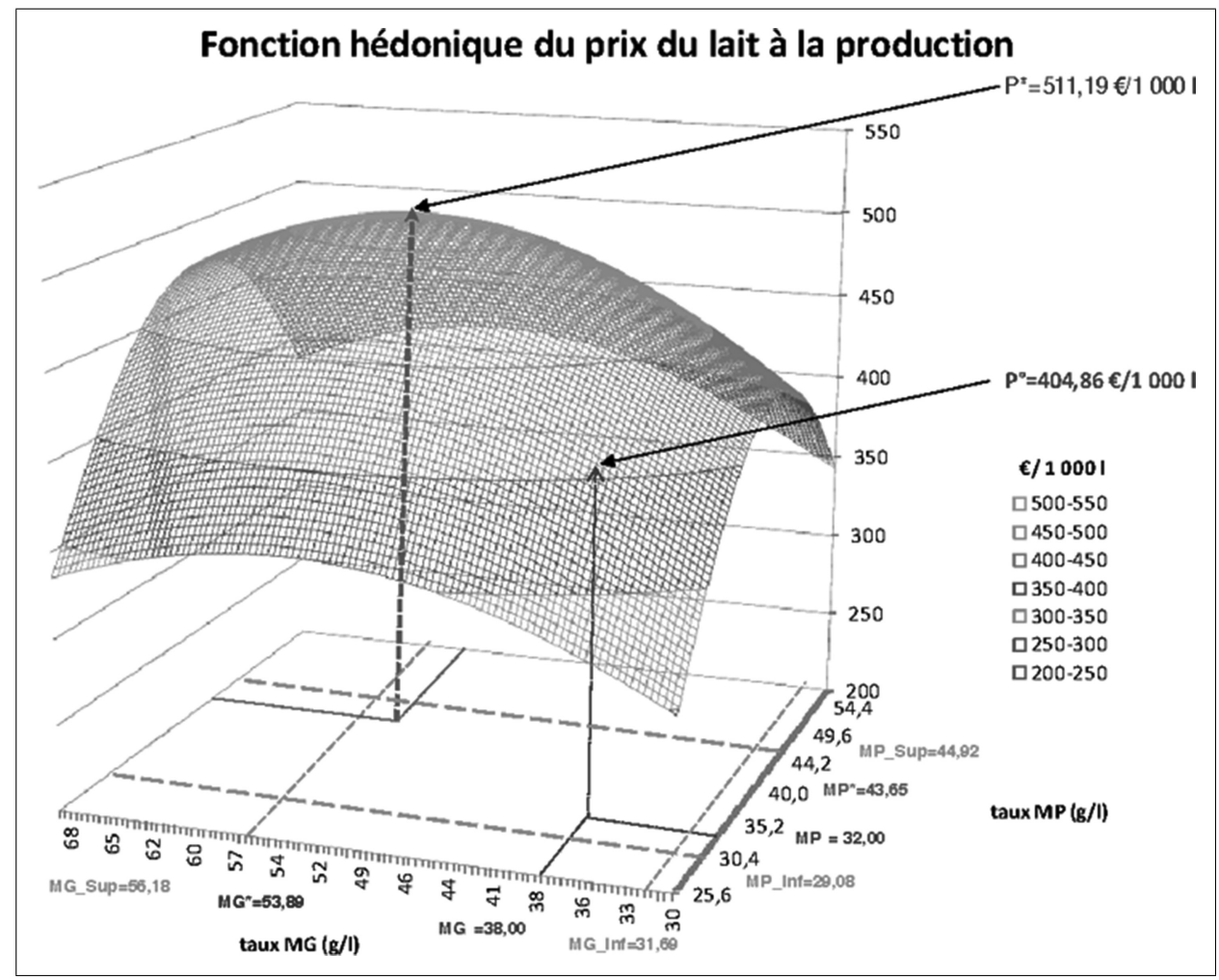

considérer comme hautement improbables pour cet horizon les valorisations marginales négatives résultant de taux comparables à ceux de la Jersiaise pour la matière grasse $(58,00 \mathrm{~g} / \mathrm{l})$ et à ceux de la Bufflonne pour la matière protéique $(46,44 \mathrm{~g} / \mathrm{l})$.

Aux effets des covariants que sont les taux de matière grasse et protéique, viennent s'ajouter ceteris paribus les effets des différents attributs qualitatifs du lait.

\subsubsection{Analyse des attributs qualitatifs du lait}

Un premier type d'attribut qualitatif a trait aux particularités de l'exploitation laitière, exprimées en termes d'appartenance à l'accord interprofessionnel national décliné selon des modalités régionales en zone CRIEL. Le différentiel propre à chaque CRIEL s'interprète relativement à l'estimation du prix du lait standard pour la Savoie pour un producteur bénéficiant de l'accord régional CRIEL. Le regroupement des zones CRIEL en trois segments de marché conduit à des effets marginaux estimés à des démarques de $132,55 € / \mathrm{Kl}$ pour Haute-Normandie et de $130,67 € / \mathrm{Kl}$ pour les autres zones $\mathrm{CRIEL}$. Le fait de 
commercialiser son lait en dehors des accords CRIEL génère une démarque de $112,97 € / \mathrm{Kl}$, proche mais significativement distincte de celle attribuée aux autres zones CRIEL. Ainsi, les prix du lait payés aux producteurs par les transformateurs hors Savoie sont bien inférieurs aux prix payés par leurs homologues de la filière savoyarde.

Les autres attributs qualitatifs sélectionnés relèvent plus directement du produit (signes de qualité), de sa commercialisation (mix-produit du transformateur, vente directe, statut coopératif). Concernant tout d'abord les signes de qualité, la production de lait sous le label certifié Agriculture Biologique génère le différentiel de valorisation le plus important en termes de niveau de prix avec un bonus marginal de 82,29 €/Kl. Par ordre d'importance dans l'effet-prix, ce signe de qualité est suivi par les signes officiels bénéficiant d'une valeur implicite de 29,02 $€ / \mathrm{Kl}$ pour l'AOC/AOP et de 9,95 $€ / \mathrm{Kl}$ pour le Label Rouge. A contrario, l'IGP génère une décote de 27,39 $€ / \mathrm{Kl}$ supérieure aux écarts " prix IGP - prix Savoie » $(-20 € / \mathrm{Kl}$, en moyenne) estimables d'après l'Enquête mensuelle laitière. Puis, le mix-produit de l'établissement laitier, « client » du producteur auprès duquel il se fournit, influe de façon significative et positivement sur le prix du lait à la production : ainsi, pour les producteurs livrant aux établissements caractérisés par un mix-produit "Fromages et produits frais », observe-t-on un bonus estimé en valeur à $11,19 € / \mathrm{Kl}$, soit $2,76 \%$ du prix de référence. Par contre, la présence de ventes directes de lait, mode plus complémentaire qu'alternatif de commercialisation, génère un bonus inférieur de $6,91 € / \mathrm{Kl}$, correspondant à $1,71 \%$ du prix de référence, en raison de la faiblesse des volumes concernés. Enfin, le statut du coopérateur génère une démarque sur le prix du lait livré par le producteur estimée à $50,81 € / \mathrm{Kl}$ qui correspond à une baisse de $12,55 \%$ du prix de référence.

\subsubsection{Analyse des interactions}

Après avoir circonscrit l'analyse des interactions aux conditions de production et de commercialisation pertinentes, nous constatons que l'effet induit par le zonage régional CRIEL est conditionné par le signe de qualité du produit laitier ainsi que le statut du producteur.

En Haute-Normandie, le zonage CRIEL interagit négativement avec la transformation en AOC/AOP avec une décote de 54,65 $€ / \mathrm{Kl}$ (13,5\% du prix de référence), ce qui en sommant les effets propres et l'interaction conduit à un effet marginal composite de $-158,19 € / \mathrm{Kl}$ pour 2006 , singulièrement contreintuitif pour les AOC/AOP de Haute-Normandie même dans un contexte de prix à la baisse.

L'interaction entre le statut de coopérateur du producteur et l'ensemble formé par les autres zones CRIEL procure un bonus estimé à 54,84 $€ / \mathrm{Kl}$ (soit $13,54 \%$ du prix de référence), ce qui amène un effet marginal composite de $-123,00 € / \mathrm{Kl}$. Le statut de coopérateur laitier interagit également avec le caractère non régulé des transactions s'opérant hors zones CRIEL conduisant à 
un bonus estimé à 10,07\% du prix de référence, soit 40,77 $€ / \mathrm{Kl}$, et un effet marginal composite évalué à $126,64 € / \mathrm{Kl}$.

Enfin, l'interaction entre le signe de qualité IGP et le mix-produit "Fromages frais et produits frais " induit un bonus estimé à 22,43 $€ / \mathrm{Kl}$ (soit $5,54 \%$ du prix de référence) qui corrige l'effet globalement dépresseur de l'IGP pour aboutir à un effet marginal composite de $6,23 € / \mathrm{Kl}$ pour 2006 , cohérent avec le niveau de valorisation des laits transformés en crème fraîche d'Alsace ou bien en Emmental et Tome de Savoie.

Ainsi, le modèle hédonique avec interactions permet d'explorer précisément le déterminisme des prix du lait spécifique aux situations de production se distinguant d'un schéma général commun (production d'un lait indifférencié assimilé à une commodité agricole). Il complète la rémunération des composants physiques (matière grasse et matière protéique) par la valorisation d'attributs qualitatifs caractérisant les situations de marché, les formes de régulation, les transformations en produits laitiers, l'organisation de la filière et les stratégies de commercialisation, en lien avec la structuration territoriale et le système des signes de qualité en vigueur.

Dans la perspective d'un approvisionnement adéquat du secteur de la transformation laitière au moyen d'une meilleure prise en compte des attributs qualitatifs spécifiant le contexte de production et de transformation laitière, l'interprétation économique de leurs effets marginaux sur le prix du lait de vache à la production est présentée dans la section suivante.

\subsection{Interprétations économiques}

Le niveau des cotations de produits laitiers industriels (beurre industriel, poudre de lait et de lactosérum) ne peut expliquer à lui seul la variabilité des prix à la production résultant des échanges entre producteurs et transformateurs, telle qu'elle nous est restituée par la «conjoncture laitière » (www.franceagrimer.fr). Pour les déterminants du prix du lait à la production sélectionnés par le modèle hédonique que nous avons estimé, nous interprétons tout d'abord les effets propres des différents facteurs puis ceux de leurs interactions.

\subsubsection{Effets propres des facteurs}

\subsubsection{Matière grasse et matière protéique}

Le modèle hédonique permet de quantifier des niveaux de rémunération qui résultent de la distribution a posteriori des différentiels de matière grasse et de matière protéique par rapport aux taux standards respectifs. Les niveaux atteints par ces bonus (4,48 et $10,10 € / \mathrm{Kl}$, respectivement) constituent des incitations dans la sélection du cheptel laitier et la conduite alimentaire de l'atelier. Le taux protéique en constitue le facteur quantitatif majeur du paiement à la qualité en raison de l'importance que revêt son amélioration pour 
la transformation fromagère : la teneur en caséine conditionne le rendement fromager lors de la coagulation. Cependant, les valorisations les plus élevées supposent de fournir également un taux de matière grasse suffisant : texture et qualités sensorielles du fromage sont conditionnées par le rapport matière grasse sur matière sèche. En outre, le taux de matière grasse détermine le rendement pour les segments supérieurs du marché en beurres et crèmes (Alsace, Normandie et Poitou-Charentes).

\subsubsection{Accords interprofessionnels régionaux}

Parmi les déterminants de la « conjoncture laitière ", la dynamique interprofessionnelle générée par les interactions entre producteurs et transformateurs joue un rôle majeur pour autant qu'on puisse en juger par les différentiels qu'elle génère entre les rémunérations consenties en Savoie (supérieures) ou en Haute-Normandie (intermédiaires), et les autres zones CRIEL (inférieures).

\subsubsection{Signes de qualité}

Nos résultats montrent également un impact significatif des signes officiels de qualité dont l'effet est positif (Agriculture Biologique, AOC/AOP et Label Rouge) à l'exception de l'IGP : il s'agit de ceux bénéficiant de la meilleure notoriété auprès du consommateur. Grâce à la valorisation des produits laitiers, les signes de qualité connus du public, tels que l'AOC/AOP et la production en Agriculture Biologique, génèrent des différentiels de rémunération appréciables que la fonction de prix hédonique permet de quantifier compte tenu du contexte régional de production. Cependant le classement selon la notoriété du signe officiel de qualité, tous produits confondus, n'implique pas une hiérarchie homothétique des consentements à payer pour les produits laitiers : ainsi, le Label Rouge, de notoriété supérieure à l'AOC $^{10}$ (Tavoularis et al., 2007), a un effet marginal inférieur à celui de l'AOC dans notre étude.

Cependant, ce bonus ne s'étend pas à tous les signes de qualité tant officiels que privé car ni la Certification de conformité de produit, ni les signes de qualité contractuels comme le label AgriConfiance et la Charte des bonnes pratiques d'élevage, ne génèrent de différences significatives. Cela confirme une analyse sur la période 2004-2006 (Desbois et Nefussi, 2007) selon laquelle ces signes, que nous qualifions de « professionnels » compte tenu du périmètre de leur notoriété, sont insuffisamment connus des consommateurs, comme dans le cas de la Certification de conformité de produit (Tavoularis et

10 Dans l'enquête CREDOC-INC de 2001, à la question «Pouvez-vous me citer tous les signes de qualité apposés sur les produits alimentaires que vous connaissez ? », $43 \%$ des Français citent le Label Rouge en premier contre $18 \%$ pour la mention Agriculture Biologique et $12 \%$ pour l'AOC. 
al., 2007), pour être valorisé dans l'échange producteur-transformateur. En effet, soit ils sont exprimés selon un référentiel de qualité trop technique pour être vraisemblablement compris du consommateur (Valceschini et Blanchemanche, 2005) soit ils se différencient trop peu de l'observation stricto sensu des dispositions réglementaires (cas de la Charte interprofessionnelle publique des bonnes pratiques d'élevage et du label coopératif privé AgriConfiance) pour impliquer un accès sélectif des producteurs laitiers aux marchés les plus rémunérateurs.

Les constats suivants peuvent expliquer la démarque de valorisation pour l'IGP : i) $40 \%$ des producteurs IGP sont situés en Alsace : ainsi, le lait livré pour l'IGP Crème fraîche fluide d'Alsace génère une rémunération inférieure à celle du lait livré pour l'AOC régionale (Munster) ; ii) 37,5\% des producteurs IGP de l'échantillon sont localisés en zone CRIEL Savoie où la décote observée confirme l'écart croissant entre le prix des laits IGP et AOC constaté en Haute-Savoie entre 1985 et 2000 (Chatellier et Delattre, 2003) résultant selon l'INAO du faible pourcentage d'IGP «fermières » (Tomme et Emmental de Savoie) par rapport aux AOC «fermières » (Reblochon et Abondance) qui tirent les prix vers le haut ; iii) $10 \%$ des producteurs IGP sont situés en Lorraine où l'Emmental Est-Central (Vosges) supporte la concurrence de l'Emmental générique produit dans l'Ouest de la France (Calvez, 2005). Les productions de l'échantillon déclarées sous IGP et ne s'affichant pas sous Label Rouge se situent principalement en zone CRIEL Savoie $(57 \%)$ et en Lorraine (19\%), ce qui confirme ces localisations comme spécifiques de la décote IGP.

\subsubsection{Mix-produit de l'établissement laitier}

Le bonus enregistré par le mix-produit «Fromages et produits frais » par rapport à un mix-produit indifférencié confirme a contrario le diagnostic sur la filière laitière française, effectué par le Comité permanent des inspections du Ministère de l'Agriculture (COPERCI, 2004), d'un risque économique pour les établissements fortement producteurs de produits industriels, démontrant ainsi l'avantage pour les producteurs de contracter avec des établissements transformateurs orientés vers les produits de grande consommation en raison des perspectives de croissance sur le marché domestique pour les produits frais, et sur les marchés d'exportation pour les fromages.

\subsubsection{Statut du producteur laitier}

Le malus important enregistré par les producteurs de lait ayant le statut de coopérateur fait écho aux difficultés de restructuration de la filière laitière coopérative (Tozanli, 2001) : la décote observée sur le lait vendu aux coopératives est-elle la marque d'un secteur qui serait handicapé dans l'adaptation de son mix-produit et de ses volumes par les règles de 
fonctionnement spécifiques à son statut ${ }^{11}$, une contribution solidaire du producteur à l'économie coopérative de la transformation laitière (Touzet, 2014), ou bien l'impact défavorable du mix-produit de coopératives centrées sur la collecte et la production de commodités industrielles à faible valeur ajoutée (COPERCI, 2004)?

\subsubsection{Interactions des facteurs}

Cependant, bonus et malus ne s'appliquent pas de façon inconditionnelle pour les déterminants sélectionnés. Les interactions entre facteurs peuvent soit renforcer soit modérer les différentiels de rémunération du lait à la production.

Au premier rang par son effet marginal figure l'interaction entre le zonage CRIEL et le signe de qualité AOC/AOP générant une démarque importante et contre-intuitive pour les laits collectés en Haute-Normandie dans les périmètres AOC de cette zone. Peu robuste car reposant sur trois observations d'une même exploitation atypique, son estimation est trop fragile pour être interprétée.

L'interaction entre le zonage CRIEL et le statut coopératif du producteur vient atténuer quelque peu la démarque pesant sur le lait des coopérateurs en Autres Zones CRIEL (bonus de 4,03 $€ / \mathrm{Kl}$ par rapport aux non-coopérateurs en Autres Zones CRIEL) alors qu'elle accentue cette démarque en Hors Zones CRIEL (malus de 10,03 $€ / \mathrm{Kl}$ par rapport aux non-coopérateurs en Hors Zones CRIEL), bien que la rémunération des coopérateurs Hors Zones CRIEL soit supérieure $(+3,64 € / \mathrm{Kl})$ à celle des coopérateurs dans l'ensemble des Autres Zones CRIEL. Ainsi, hormis la Savoie, alors que les accords CRIEL ne s'avèrent pas favorables aux producteurs de lait par rapport au marché hors zones (malus de $19,58 € / \mathrm{Kl}$ pour la Haute-Normandie et de $17,70 € / \mathrm{kl}$ pour les Autres Zones CRIEL), ce bonus de $4,03 € / \mathrm{Kl}$ indique néanmoins que la régulation CRIEL protège quelque peu les coopérateurs dans les contextes les moins favorables de valorisation.

L'interaction positive de l'IGP avec le mix-produit orienté « Fromages et produits frais » fait plus que compenser $(+33,62 \epsilon / \mathrm{Kl})$ le malus marginal affecté à l'IGP pour des producteurs contractant avec des transformateurs au mix-produit différencié : en l'occurrence, il s'agit essentiellement (pour $89 \%$ ) de producteurs alsaciens livrant à un transformateur spécialisé en produits frais de qualité (Crème fraîche fluide d'Alsace) et assez minoritairement (pour $11 \%$ ) d'éleveurs lorrains livrant pour la transformation fromagère (Emmental Est-Central vosgien).

11 Par exemple, la coopérative laitière a l'obligation de collecter tout le lait de ses adhérents qui en retour lui doivent un apport total de leur lait. N'ayant pas cette obligation, les transformateurs privés peuvent arrêter définitivement la collecte en arguant de motifs économiques. En 2012, de nombreuses ruptures de collecte ont été enregistrées pour ces motifs ( $c f$. communiqué de la Fédération nationale des producteurs laitiers, 2 juillet 2012). 


\section{Discussion et conclusions}

Le modèle hédonique des prix du lait à la production étudié dans cet article permet d'estimer les valeurs implicites des attributs tant quantitatifs que qualitatifs du lait à la production dans une période de croissance des prix. Outre les valeurs marginales des taux de matière grasse et de matière protéique, nous fournissons les valeurs implicites des attributs qualitatifs du lait à la production relevant soit du contexte de production (signes de qualité), de commercialisation (zone CRIEL, statut de coopérateur, vente directe), ou de transformation (mix-produit du transformateur).

Grâce au contrôle laitier, les établissements laitiers sont bien informés sur la composition de l'ensemble des laits produits dans leur aire de collecte. Ainsi, le niveau d'information des établissements laitiers ne semble pas trop éloigné de l'hypothèse d'information parfaite pour invalider la démarche d'analyse. Cependant, le concept d'équilibre hédonique suppose que les producteurs de lait soient également informés des prix spécifiques aux différents types de lait y compris ceux qu'ils auraient décidé de ne pas produire, compte tenu des facteurs de production dont ils disposent et du coût de production induit par ces types de lait. De ce point de vue, une certaine asymétrie d'information persiste entre organisations professionnelles de producteurs laitiers et établissements laitiers (Kroll et al., 2010), en dépit des efforts de l'interprofession (Emorine et Bailly, 2009). En outre, la nature concurrentielle du marché français du lait à la production pour la période considérée a été mise en question au regard de la réglementation communautaire (Conseil de la concurrence, 2007).

Une modélisation hédonique modélisation hédonique complète supposerait d'analyser la position concurrentielle des transformateurs laitiers vis-à-vis des opérateurs de la grande distribution, à l'instar de Mérel (2009) pour le gruyère de Comté. Cependant, cela impliquerait de collecter l'ensemble des données empiriques permettant de tester la pertinence des différents régimes concurrentiels.

Aussi, sur la base de la fonction hédonique du prix du lait à la production, nous concluons à une structuration du marché français du lait en quatre segments (Haute-Normandie, Autres Zones CRIEL, Hors Zones CRIEL et Savoie), à une meilleure rémunération de la matière protéique relativement à la matière grasse, à un bonus rémunérateur pour certains signes de qualité officiels (Agriculture Biologique, AOC/AOP, Label Rouge), et enfin à l'influence significativement positive d'un mix-produit spécifique du transformateur orienté vers la transformation fromagère et les produits frais. Cependant, le bonus apporté par le signe de qualité officiel peut être conditionné par le mix-produit du transformateur comme pour l'IGP ou non significatif comme pour la Certification de conformité de produit. En outre, les signes de qualité professionnels comme le label AgriConfiance et la Charte des bonnes pratiques d'élevage, ne génèrent pas de différences significatives. Même s'ils s'avèrent plus faibles en termes d'impact sur les prix à la production, l'effet 
attribuable au mix-produit de l'établissement laitier apporte une information pertinente pour l'analyse de la chaîne de valeur des produits laitiers, l'orientant résolument vers les fromages, affinés ou frais, et les produits frais.

Les différences de valorisation implicite constatées s'interprètent en termes de maîtrise des signes officiels de qualité par le producteur, de pouvoir de négociation de la rente territoriale, de niveau concurrentiel des segments de marché suprarégionaux, et de déterminants économiques favorables au maintien de la production laitière de qualité sur certains territoires, comme pour le Comté, ou a contrario moins favorables, comme pour le Cantal (Desquilbet et Monier-Dilhan, 2015). Cependant ces différentiels de rémunération dépendent non seulement de la localisation de la production au plan territorial mais également au plan sectoriel de l'organisation de la filière de production. En effet, si le différentiel de prix constaté s'analyse en termes de rente territoriale pour l'AOC/AOP (Mollard et al., 2001), il s'interprète pour l'Agriculture Biologique et le Label Rouge en termes de rente organisationnelle générée par des formes spécifiques de coordination émergeant au croisement du sectoriel et du territorial (par ex., le système des fruitières en Comté) sous forme de systèmes agroalimentaires localisés (Muchnik et al., 2007). Certifiée indépendamment de l'aval de la production, la rente générée par le signe Agriculture Biologique est supérieure à celle des autres signes officiels ; parmi ceux-ci, la rente majeure revient à l'AOC/AOP, dont le lien au territoire est le mieux affirmé. Le déficit de l'IGP (signe européen) est attribuable à un lien territorial plus lâche par rapport à l'AOP/AOC strictement délimitée et à une moindre notoriété par rapport au Label Rouge (signe français) bénéficiaire d'une grande antériorité.

Les résultats obtenus traduisent également l'importance stratégique prise par les produits de grande consommation, dont témoigne l'émergence des marques de distributeurs. Sans que le modèle de transformation indifférenciée en produits industriels soit complètement remis en cause pour certains opérateurs, il apparaît crucial pour la filière de tenir compte de ces signaux de marché, valorisant la transformation en fromages et produits frais dans les systèmes de rémunération de l'après-suppression des quotas laitiers. Dans cet univers de produits laitiers où les signes de qualité proliferent, l'Agriculture Biologique et l'AOC/AOP constituent des valeurs éprouvées, potentiellement synergiques pour des produits gastronomiques. Cependant, comme le montre l'interaction de l'IGP avec le mix-produit du transformateur, la quote-part rentière des signes officiels de qualité concédée au producteur apparaît conditionnée par la maîtrise du processus de commercialisation du lait et les opportunités de transformation que peuvent garantir organisations de producteurs et syndicats de transformateurs, face aux opérateurs de la distribution dont l'influence est devenue majeure sur les marchés de consommation des produits laitiers sous signes de qualité (Hassan et Monier-Dilhan, 2006).

Sur la base de ces estimations hédoniques, nous montrons qu'il y a un avantage-prix pour les producteurs laitiers à contractualiser avec des 
filières de transformation dont les produits s'inscrivent dans une démarche de différenciation des produits, adossée aux signes de qualité officiels des produits laitiers et, lorsque cela est possible, du lait lui-même par la création de mentions valorisantes au niveau européen.

Compte tenu des objectifs de maîtrise des coûts et de réorientation du mix-produit (des produits industriels vers les produits de grande consommation), l'hypothèse que les différences non-significatives parmi les zones CRIEL puissent s'accentuer avec la suppression du régime des quotas laitiers en 2015, soit en raison de systèmes régionaux spécifiques fondés sur le lien entre les produits et le territoire (zones CRIEL Savoie, Rhône-Alpes, Haute-Normandie, Basse-Normandie, Auvergne-Limousin) soit selon la dynamique interprofessionnelle de concentration et de restructuration du secteur laitier (zones CRIEL Bretagne et Pays-de-Loire, Nord-Picardie-Ardennes, Sud-Ouest), devra être examinée. Du fait de son engagement à amortir les fluctuations de prix et de volume dans ses approvisionnements, le secteur coopératif laitier serait alors conforté dans une stratégie tant de concentration de collecte sur les bassins de grande densité laitière à flexibilité productive négociable que de développement des capacités de production pour les produits de grande consommation génériques à plus grande valeur ajoutée.

\section{Remerciements}

Cette recherche s'appuie sur la collecte de données comptables cofinancée par les conventions CNIEL-SCEES/MAP 2006, 2007 et 2008 portant sur la Sonde laitière du RICA (SSP/Agreste). Seul responsable du contenu de cet article ainsi que des éventuelles erreurs ou omissions, l'auteur remercie pour leur soutien et/ou leur conseil le Centre National Interprofessionnel de l'Economie Laitière (CNIEL), le Secrétariat général du Ministère de l'Agriculture, JeanChristophe BUREAU, Emmanuel CHANTRY, Sylvette MONIER-DILHAN et Yves SURRY, ainsi que les deux relecteurs anonymes de RAEStud. L'auteur dédie ce travail à la mémoire de son père, un des précurseurs de l'élevage "bio" en race mixte Maine-Anjou devenue l'AOP Rouge des Prés depuis 2004.

\section{Bibliographie}

Aubert M., Perrier-Cornet P. (2009) Is there a future for small farms in developed countries? Evidence from the French case, Agricultural Economics 40(s1), 797-806.

Bouamra-Mechemache Z., Chavas J. P., Cox T., Réquillart V. (2002) Partial market liberalization and the efficiency of policy reform: the case of the European dairy sector, American Journal of Agricultural Economics 84(4), 1003-1020. 
Calvez E. (2005) Un récent « déménagement » du territoire fromager français : l'exemple de l'emmental, L'information géographique 69(2), 184-194.

Chatellier V. et Delattre F. (2003) La production laitière dans les montagnes françaises : une dynamique particulière pour les Alpes du Nord, INRA Productions Animales 16(1), 61-76.

Chatellier V., Lelyon B., Perrot C. et You B. (2013) Le secteur laitier français à la croisée des chemins, INRA Productions Animales 26(2), 77-100.

Conseil de la concurrence (2007) Décision n 07-D-10 du 28 mars 2007 relative à une plainte à l'encontre du Comité interprofessionnel du gruyère de Comté, URL : http://www.autoritedelaconcurrence.fr/pdf/avis/07d10.pdf, 18 p.

COPERCI (2004) Comité permanent de coordination des inspections du ministère de l'Agriculture, de l'alimention, de la Pêche et des affairs rurales, L'avenir de la filière laitière française, Trédé Yves (ed), Réf. 2089, Paris, France, 92 p.

Desbois D. et Nefussi J. (2007) Signes de qualité : prix du lait et rentabilité des exploitations, Agreste Cabiers 3, 3-18.

Desquilbet M. et Monier-Dilhan S. (2015) Are geographical indications a worthy quality label? A framework with endogenous quality choice, European Review of Agricultural Economics 42(1), 129-150.

Ekeland I., Heckman J, Nesheim L. (2004) Identification and estimation of hedonic models, Journal of Political Economy 112(s1), S60-S109.

Emorine J-P. et Bailly G. (2009) Avis de l'Autorité de la concurrence relative au fonctionnement du secteur laitier, Rapport du Sénat $\mathrm{n}^{\circ} 73$, Paris, France, $82 \mathrm{p}$.

Gillmeister W. J., Yonkers R. D. et Dunn J. W. (1996) Hedonic pricing of milk components at the farm level, Review of agricultural Economics 18(2), 181-192.

Grivaux J. (2008) Enquête sur la structure des exploitations agricoles en 2007 : 326000 exploitations professionnelles, Agreste Primeur 215, octobre, $4 \mathrm{p}$.

Halvorsen R. et Pollakowski H.O. (1981) Choice of functional form for hedonic price equations, Journal of Urban Economics 10(1), 37-49.

Hassan D. et Monier-Dilhan S. (2002) Signes de qualité et qualité des signes : une application au marché du camembert, Cabier d'Economie et Sociologie Rurales 65, 23-36.

Hassan D. et Monier-Dilhan S. (2006) National brands and store brands: competition through public quality labels, Agribusiness 22(1), 21-30.

Idele (2014) Institut de l'élevage, Le cheptel laitier français. Evolution génétique et phénotypique 1993-2013 - Prévision d'évolution génétique 2013-2019, Compte-rendu n ${ }^{\circ} 0014200017$, Paris, France, 40 p. 
Kennedy P. (1981) Estimation with correctly interpreted dummy variables in semilogarithmic equations, American Economic Review 71(4), 801.

Kroll J.-C., Trouvé A., Déruaz M. (2010) Quelle perspective de régulation après la sortie des quotas? Faut-il encore une politique laitière européenne?, Rapport CESAER, INRA-Agrosup, $42 \mathrm{p}$.

Ladd G. W. et Martin M. B. (1976) Prices and demands for input characteristics, American Journal of Agricultural Economics 58(1), 21-30.

Ladd G. W. et Suvannunt V. (1976) A model of consumer goods characteristics, American Journal of Agricultural Economics 58(3), 504-510.

Lagrange L., Briand H. et Trognon L. (2000) Importance économique des filières agro-alimentaires de produits sous signes officiels de qualité [Etude comparée de leur évolution en France et en UE], Economie Rurale 258, 6-18.

Liu D. J., Kaiser H. M., Forker O. D., Mount T. D. (1990) An economic analysis of the U.S. generic dairy advertising program using an industry model, Northern Journal of Agricultural and Resource Economics 19(1), 3748.

Marette S. (2009) Quels instruments économiques de régulation de la qualité ? Marchés et réglementation dans le secteur agro-alimentaire, Inra Sciences Sociales 1, $4 \mathrm{p}$.

Mallows C. L. (1973) Some comments on $C_{p}$, Technometrics 15(4), 661-675.

Mérel P. (2009) Measuring market power in the French Comté cheese market, European Review of Agricultural Economics 36(1), 31-51.

Mollard A., Pecqueur B. et Lacroix A. J. (2001) A meeting between quality and territorialism: the rent theory reviewed in the context of territorial development, International Journal of Sustainable Development 4(4), 368371 .

Muchnik J., Requier-Desjardins D., Sautier D. et Touzard J.-M. (2007) Systèmes agroalimentaires localisés, Economie et Sociétés AG 29, 1465-1484.

Rosen S. (1974) Hedonic prices and implicit markets: product differentiation in pure competition, Journal of Political Economy 82(1), 34-55.

Tavoularis G., Recours F. et Hebel P. (2007) Perception de la qualité et des signes officiels de qualité dans le secteur alimentaire, Cabier de Recherche Credoc 236, $114 \mathrm{p}$.

Touzet C. (2014) Systèmes de régulation et solidarité pour les filières laitières biologiques, Economie rurale 339-340, 49-63.

Tozanli S. (2001) La filière laitière en France : l'évolution de l'industrie de la transformation pendant ces vingt dernières années, CIHEAM Options méditerranéennes, série B, Etudes et recherches 32, 171-194. 
Valceschini E. (2008) Les produits d'origine dans l'économie de la qualité : quelles évolutions, communication, $X^{\mathrm{e}}$ Assises de l'Origine, Bordeaux, France, 6 mai 2008.

Valceschini E. et Blanchemanche S. (2005) La certification de conformité de produits sur les marchés agroalimentaires : différenciation ou normalisation?, Notes et études économiques 24, 7-40.

White H. (1980) A heteroskedasticity-consistent covariance matrix estimator and a direct test for heteroskedasticity, Econometrica 48(4), 817-838.

Zhu Y., Cox T. et Chavas J-P. (1999) An economic analysis of the effects of the Uruguay Round Agreement and full trade liberalization on the world dairy sector, Canadian Journal of Agricultural Economics 47(5), 187-200. 